\title{
ORIGIN OF ULTRA-HIGH-ENERGY GALACTIC COSMIC RAYS: THE ISOTROPY PROBLEM
}

\author{
Martin Pohl ${ }^{1,2}$ and David Eichler ${ }^{3}$ \\ ${ }^{1}$ Institut für Physik und Astronomie, Universität Potsdam, 14476 Potsdam-Golm, Germany \\ ${ }^{2}$ DESY, 15738 Zeuthen, Germany \\ ${ }^{3}$ Physics Department, Ben-Gurion University, Be'er-Sheva 84105, Israel \\ Received 2011 April 22; accepted 2011 August 9; published 2011 November 15
}

\begin{abstract}
We study the propagation of ultra-high-energy cosmic rays (UHECRs) in the Galaxy, concentrating on the energy range below the ankle in the spectrum at $4 \mathrm{EeV}$. A Monte Carlo method, based on analytical solutions to the time-dependent diffusion problem, is used to account for intermittency by placing sources at random locations. Assuming a source population that scales with baryon mass density or star formation (e.g., long GRB), we derive constraints arising from intermittency and the observational limits on the composition and anisotropy. It is shown that the composition and anisotropy at $10^{18} \mathrm{eV}$ are difficult to reproduce and require that either (1) the particle mean free path is much smaller than a gyroradius, implying the escape time is very long, (2) the composition is heavier than suggested by recent Auger data, (3) the ultra-high-energy sub-ankle component is mostly extragalactic, or (4) we are living in a rare lull in the UHECR production, and the current UHECR intensity is far below the Galactic time average. We therefore recommend a strong observational focus on determining the UHECR composition around $10^{18} \mathrm{eV}$.
\end{abstract}

Key words: cosmic rays - gamma-ray burst: general

Online-only material: color figures

\section{INTRODUCTION}

An open problem in cosmic-ray (CR) astrophysics is at what energy we observe the transition from a Galactic to an extragalactic CR origin, and what sorts of sources, in and out of our own Galaxy, are responsible for each population.

It is widely suspected that the abrupt flattening of the spectrum at $E=4 \times 10^{18} \mathrm{eV}$, the so-called ankle of the CR spectrum, indicates the Galactic to extragalactic transition, but the abrupt increase in loss rate above the ankle due to pair production with the microwave background may also play a role. It is generally recognized that blast waves from supernova remnants (SNR) cannot accelerate $\mathrm{CR}$ protons beyond energies of about $10^{14.5} \mathrm{eV}$ (Lagage \& Cesarsky 1983), so they could not reach the CR ankle, unless the Galactic magnetic field is amplified by the blast wave by many orders of magnitude. This motivated Levinson \& Eichler (1993) to propose that ultra-high-energy cosmic rays (UHECRs) are produced by gamma-ray bursts (GRBs) that occur in our Galaxy.

This is the third paper of a series, earlier publications of which demonstrated that (1) long GRBs likely have insufficient power to provide UHECRs at energies above the observed ankle at $4 \times 10^{18} \mathrm{eV}$ (Eichler et al. 2010), unless their CR power is much higher than their $\gamma$-ray power, and (2) at energies below the ankle the contribution from Galactic GRB should on average be stronger by about three orders of magnitude than that of extragalactic GRBs (as the latter would imply that extragalactic space is filled uniformly), thus further constraining models involving a GRB origin of transankle particles (Eichler \& Pohl 2011, henceforth referred to as Paper II).

In this paper, we study the time-dependent diffusive transport of UHECRs in the Galaxy using the method of Monte Carlo to account for the unknown location and explosion time of GRB or other sources with similar population statistics. For simplicity, we shall call the sources GRBs throughout the paper, but it is to be understood that the study applies to other classes of UHECR sources as well (e.g., $\gamma$-ray silent hypernovae). Our approach permits us to quantitatively account for intermittency effects in the local UHECR spectrum and thus goes beyond the scope of earlier publications (Levinson \& Eichler 1993; Wick et al. 2004; Calvez et al. 2010). Faced with the observed energy spectrum and low limits on anisotropy recently set by Auger and other experiments, we calculate spectra and anisotropy that would be expected as a function of model parameters. The ceiling on anisotropy, of order $1 \%$ or less below $10^{18} \mathrm{eV}$, is interesting given the rather large mean free paths of such energetic cosmic rays.

We assume the propagation in the Galaxy of cosmic rays at energies $10^{15}-10^{18} \mathrm{eV}$ can be accurately described as isotropic diffusion. The framework of isotropic diffusion requires that the particle Larmor radii be considerably smaller than the dimensions over which the diffusion is considered. If this were not the case, our conclusion that isotropy is hard to understand would be strengthened, as there would be little chance of isotropizing UHECRs emerging from an anisotropic source distribution. Likewise, the particle mean free path, $\lambda_{\mathrm{mfp}}$, should be much smaller than a few kpc, the typical distance between the solar system and a GRB in the Galaxy. The Larmor radius of a $Z=1$ particle in a $10 \mu \mathrm{G}$ field is used as a scale in this paper. It reaches $\sim 100 \mathrm{pc}$ at $10^{18} \mathrm{eV}$, and therefore the first approximation should hold for UHECRs of any composition below $\approx 10^{18} \mathrm{eV}$. The second approximation requires that $\lambda_{\mathrm{mfp}}$ be within a factor of $\sim 10$ of the Larmor radius. Aloisio et al. (2009) have studied the transition from the rectilinear regime to the diffusive regime of particle propagation, concluding that diffusion models are a good approximation if the source distance is $L>6 \lambda_{\mathrm{mfp}}$. In the models presented in Section 3, for protons at $E=4 \mathrm{EeV} \lambda_{\mathrm{mfp}} \leqslant 500 \mathrm{pc}$, and it is smaller by a factor $1 / Z$ for heavy nuclei, whereas the GRB rate is so low that typically $L \gtrsim 5 \mathrm{kpc}$. As we shall demonstrate, reproducing the observed low anisotropy in the EeV band requires a mean free path small enough to maintain validity of the diffusion approach, at least for GRBs. 
Isotropic diffusion requires that the magnetic field is weakly ordered. Measurements of Faraday rotation indicate that the strength of the ordered field is about $2 \mu \mathrm{G}$, whereas the random field has an amplitude of $\sim 5 \mu \mathrm{G}$ on scales of $10-100 \mathrm{pc}$ (Han 2009, and references therein). Note that UHECRs sample the turbulent field in the spectral band where they are strongest, on the scales of energy injection by supernovae ( $\mathrm{SNe}$ ) and stellar winds.

To evaluate the level of systematic uncertainties in our model description, we explore various geometric forms of the propagation volume of UHECRs in the Galaxy. We find that a disk-like geometry, which appears more likely to be accurate than the assumption of spherical symmetry, renders the observational constraints on anisotropy and composition more difficult to meet, and resolution of this problem could lead to interesting or unconventional conclusions.

\section{PROPAGATION OF UHECRs FROM GALACTIC GRBs}

\subsection{Spherical Symmetry}

Treating energy losses as a catastrophic loss term with loss time $T$, assuming the particles are instantaneously released at time $t_{0}=0$ by a single point source, and assuming a large cosmic-ray halo, the problems are spherically symmetric with respect to the GRBs, and the differential density of UHECRs obeys a continuity equation that can be written as

$$
\frac{\partial N}{\partial t}+\frac{N}{T}-\frac{1}{r^{2}} \frac{\partial}{\partial r}\left(r^{2} D \frac{\partial N}{\partial r}\right)=Q(E) \delta(t) \frac{\delta(r)}{4 \pi r^{2}} .
$$

Here, $D=c \lambda_{\text {mfp }} / 3$ is the spatial diffusion coefficient, $r$ is the distance from the GRB, and $Q(E)$ is the differential production rate of UHECRs in a GRB. We assume that we are located outside of the dissipation region of the GRBs, implying that the plasma no longer streams with significant velocity. If we were, the Compton-Getting anisotropy would be prohibitively large. Once the GRB outflow turns non-relativistic, the further dynamical evolution is not unlike that of an SNR, and we can expect the GRB remnant to be of order $100 \mathrm{pc}$ in size, small enough to render it highly unlikely that we reside inside. The high-energy particles escaping from a GRB can initially have a rather directional distribution which leads to well-known instabilities (e.g., Medvedev \& Loeb 1999; Pohl \& Schlickeiser 2000) that provide for a rapid isotropization of the particles. We assume that this isotropization is achieved on a scale small compared with the average distance to GRBs, and hence we can approximate it as being instantaneous.

Note that Equation (1) contains the particle energy, $E$, only parametrically; it is not a variable. The catastrophic loss term permits using Dirichlet boundary conditions $N(r=\infty)=0$.

Using standard methods (Kardashev 1962; Ginzburg \& Syrovatskii 1964) one derives a general solution to the transport equation,

$$
N(r, t, E)=\exp \left(\frac{-t}{T}\right) \frac{\Theta(t)}{(4 \pi D t)^{3 / 2}} Q(E) \exp \left(-\frac{r^{2}}{4 D t}\right) .
$$

If more than one GRB contribute to the local UHECR flux at any time, their individual contributions must be calculated using Equation (2) and then summed.

The anisotropy in the UHECR intensity mainly arises from the diffusive flux (e.g., Schlickeiser 1989) and is of the order

$$
\delta=\frac{I_{\max }-I_{\min }}{I_{\max }+I_{\min }} \simeq \lambda_{\mathrm{mfp}} \frac{1}{N_{\text {tot }}}\left|\vec{\nabla} N_{\text {tot }}\right|
$$

where $N_{\text {tot }}$ is the total UHECR spectrum after summing the contributions of all relevant GRB. If there was only one GRB, then the anisotropy would be

$$
\delta \simeq \frac{3 r}{2 c t}
$$

and therefore, setting for example $r=r_{\odot}$, a given anisotropy at the solar circle, $\delta$, requires that the age of the UHECR population, $t$, obeys

$$
t \simeq \frac{3 r_{\odot}}{2 c \delta}=\left(4 \times 10^{6} \mathrm{yr}\right)\left(\frac{\delta}{10^{-2}}\right)^{-1} .
$$

At that time, the Galactic UHECRs fill a sphere of radius

$$
R \simeq \sqrt{4 D t} \simeq(10 \mathrm{kpc})\left(\frac{\lambda_{\mathrm{mfp}}}{100 \mathrm{pc}}\right)^{1 / 2}\left(\frac{\delta}{10^{-2}}\right)^{-1 / 2}
$$

To be noted from the approximation represented by Equations (4) and (6) is that the UHECR flux at the solar circle depends on the mean free path, but the anisotropy does not (e.g., Wick et al. 2004).

The above conclusion is highly significant. It means that at increasingly high energies the anisotropy may be reduced, given a sufficiently long time since the last point-source injection, to within the tight observational constraints even at energies high enough that the mean free path is a significant fraction of the geometric scale of the Galaxy, as observations indicate. We therefore investigate how the solution generalizes to arbitrary radial dependence of the diffusion coefficient.

Consider, then, Equation (4) with a radially dependent diffusion coefficient $D=D_{o} r^{j}$, with $j>0$

$$
\frac{\partial N}{\partial t}+\frac{N}{T}-\frac{1}{r^{2}} \frac{\partial}{\partial r}\left(r^{2} D_{o} r^{j} \frac{\partial N}{\partial r}\right)=Q(E) \delta(t) \frac{\delta\left(r-r_{o}\right)}{4 \pi r^{2}} .
$$

Note that we choose a finite radius of injection, $r_{o}$, in a way that preserves spherical symmetry, so that the diffusion coefficient at the injection site is finite. For $j<2$, a finite boundary condition at the origin and a vanishing boundary condition at $r=\infty$, the solution at large times (or $r \gg r_{0}$ ) approaches

$$
\begin{aligned}
N(r, t, E) \simeq & \exp \left(\frac{-t}{T}\right) \frac{\Theta(t)}{\left(4 \pi D_{o} r_{o}^{j} t\right)^{3 /(2-j)}} Q(E) \\
& \times \exp \left(-\frac{r^{2}}{(j-2)^{2} D_{o} r^{j} t}\right) .
\end{aligned}
$$

By comparison with Equation (2), the case of constant diffusion coefficient, the solution falls off more quickly after reaching a peak flux at $r^{2}=3(2-j) D_{0} r^{j} t$, whereas the anisotropy, when estimated as in Equation (3), is

$$
\delta=\frac{3 r}{(2-j) c t}
$$

(which differs from the expression in Equation (4) by the numerical factor of order unity $\left.\left(1-\frac{j}{2}\right)\right)$. That the anisotropy has similar time dependence, while the decline in intensity has steeper time dependence, has further significance: it means that to reduce the anisotropy with time at the observer's location, a larger reduction in intensity is required for positive $j$ than for $j=0$ (the case of spatially homogeneous diffusion). 
If we were to attempt accommodating the low observed UHECR anisotropy with an anisotropic source distribution by invoking an intermittent source of UHECRs followed by a period of zero source activity, we would have to pay a larger cost in intensity reduction. The intensity at Earth at the time of maximum intensity, we would conclude, would be higher than the current intensity by an even larger factor than in the case of homogeneous diffusion.

When $j>2$ the exponential factor does not vanish at $r=\infty$, and the correct Green's function at large time is

$$
\begin{aligned}
N(r, t, E)= & \exp \left(\frac{-t}{T}\right) \frac{\Theta(t)}{\left(4 \pi D_{o} r_{o}^{j} t\right)^{(1-2 j) /(2-j)}} \\
& \times Q(E)\left(r / r_{o}\right)^{-j-1} \exp \left(-\frac{r^{2}}{(j-2)^{2} D_{o} r^{j} t}\right) .
\end{aligned}
$$

In this case, the scale height of $N$ at radius $r,(d \ln N / d r)^{-1}$, is of order $r$, even at large $t$, and the anisotropy does not become arbitrarily small in the limit of large $t$. If the magnetic field of the Galaxy is dragged out by a Galactic wind, it is quite reasonable to suppose that the magnetic field decreases as $1 / r^{2}$, and that the gyroradius and mean free path increase at least as fast as $r^{2}$. It is therefore not clear whether the small observational upper limit on anisotropy can be accommodated with a realistic magnetic field geometry, no matter how intermittent the sources. Similar remarks would apply if convection were significant as compared to diffusion. Moreover, we have not yet taken into account the fact that a single intermittent source of cosmic rays would be localized in angle as well as radius, which would further increase the anisotropy.

Below we reconsider the anisotropy question assuming a freeescape boundary up to several kpc above the Galactic disk following the consideration of several other issues. The freeescape boundary models the effect of a very strong dependence of $D$ on $r$ (e.g., due to a weaker magnetic field) and/or the outward convective effects of a Galactic wind or of Galactic chimneys beyond some region. We show that the same principle applies as demonstrated above in the spherically symmetric case with spatially varying coefficient: that the leakage out the faces of the disk greatly reduces the intensity of the UHECRs as they isotropize relative to the observer; in fact, while the anisotropy declines algebraically with time, as in the previous examples, the intensity declines exponentially, so the isotropization carries a large cost in intensity reduction. This poses constraints on any theory of their origin from sources that are distributed anisotropically in the observer's sky.

\subsubsection{Heavier Nuclei}

Heavier nuclei have a smaller rigidity than protons at the same total energy, $R \propto E / Z$. The mean free path of an ultrahigh-energy particle should only depend on the rigidity, and in the absence of energy losses a nucleus of charge $Z$ and energy $E_{Z}$ should behave like a proton of energy $E=E_{Z} / Z$. Thus, Equation (2) also describes the distribution of heavy nuclei in the Galaxy, provided the appropriate scaling is applied to the energy and the source rate.

\subsection{Diffusion in Disk Geometry}

Cosmic-ray studies at lower energies indicate that a few $\mathrm{kpc}$ out into the halo diffusive transport become much faster, and particles efficiently escape from the Galaxy. For a cosmicray source near, e.g., the Galactic Center we therefore expect deviations from spherical symmetry, and a thin-disk geometry appears better suited to describe the transport of cosmic rays. The cosmic-ray continuity equation (1) then changes to

$$
\begin{aligned}
\frac{\partial N}{\partial t} & +\frac{N}{T}-\frac{\partial}{\partial x}\left(D \frac{\partial N}{\partial x}\right)-\frac{\partial}{\partial y}\left(D \frac{\partial N}{\partial y}\right) \\
& -\frac{\partial}{\partial z}\left(D \frac{\partial N}{\partial z}\right)=Q(E) \delta(z) \delta(t) q(x, y),
\end{aligned}
$$

where the halo boundary can be incorporated through boundary conditions $N(z= \pm H)=0$. Integrating over $z$ yields

$$
\begin{array}{r}
\frac{\partial M}{\partial t}+\frac{M}{T}-\frac{\partial}{\partial x}\left(D \frac{\partial M}{\partial x}\right)-\frac{\partial}{\partial y}\left(D \frac{\partial M}{\partial y}\right) \\
-\left.\left(D \frac{\partial N}{\partial z}\right)\right|_{-H} ^{H}=Q(E) \delta(t) q(x, y),
\end{array}
$$

where

$$
M=\int_{-H}^{H} d z N
$$

Instead of a computationally expensive full solution (Büsching et al. 2005), we use a steady-state solution to estimate the relation between $M$ and the mid-plane cosmic-ray density, $N_{0}$, as well as turn the diffusive flux at the halo boundaries into a simple catastrophic loss term. In the energy band of interest, escape is the dominant loss process of cosmic rays in the Galaxy, and in the steady state we expect their density to follow

$$
N(z)=N_{0}\left(1-\frac{|z|}{H}\right)
$$

implying

$$
M=H N_{0} \quad \text { and }-\left.\left(D \frac{\partial N}{\partial z}\right)\right|_{-H} ^{H}=\frac{2 D}{H^{2}} M .
$$

The diffusive escape can thus be described using a loss time

$$
\tau_{\mathrm{esc}}=\frac{H^{2}}{2 D} \simeq\left(1.2 \times 10^{6} \mathrm{yr}\right)\left(\frac{H}{5 \mathrm{kpc}}\right)^{2}\left(\frac{\lambda_{\mathrm{mfp}}}{0.1 \mathrm{kpc}}\right)^{-1}
$$

which is far shorter than the energy-loss time, $T$, which is $\gtrsim 10^{9}$ years for photo-secondary production in the Galaxy (Berezinskii \& Grigoreva 1988) and $\sim 10^{8}$ years for secondary production in p-ISM collisions (Huang et al. 2007, with average interstellar-matter density $n_{\mathrm{ISM}}<0.2$ atoms $\mathrm{cm}^{-3}$ ), and therefore energy losses can be ignored henceforth. Ignoring variations in the diffusion coefficient within the Galactic plane, the problem only depends on the in-plane distance between source (GRB) and observer, $\rho$, and can be recast as a two-dimensional diffusion equation for the mid-plane cosmic-ray density around a point source

$$
\frac{\partial N_{0}}{\partial t}+\frac{N_{0}}{\tau_{\mathrm{esc}}}-\frac{1}{\rho} \frac{\partial}{\partial \rho}\left(\rho D \frac{\partial N_{0}}{\partial \rho}\right)=Q(E) \delta(t) \frac{\delta(\rho)}{2 \pi \rho H}
$$

with solution

$$
N_{0}(\rho, t, E)=\exp \left(-\frac{t}{\tau_{\mathrm{esc}}}\right) \frac{\Theta(t)}{4 \pi D t} \frac{Q(E)}{H} \exp \left(-\frac{\rho^{2}}{4 D t}\right) .
$$




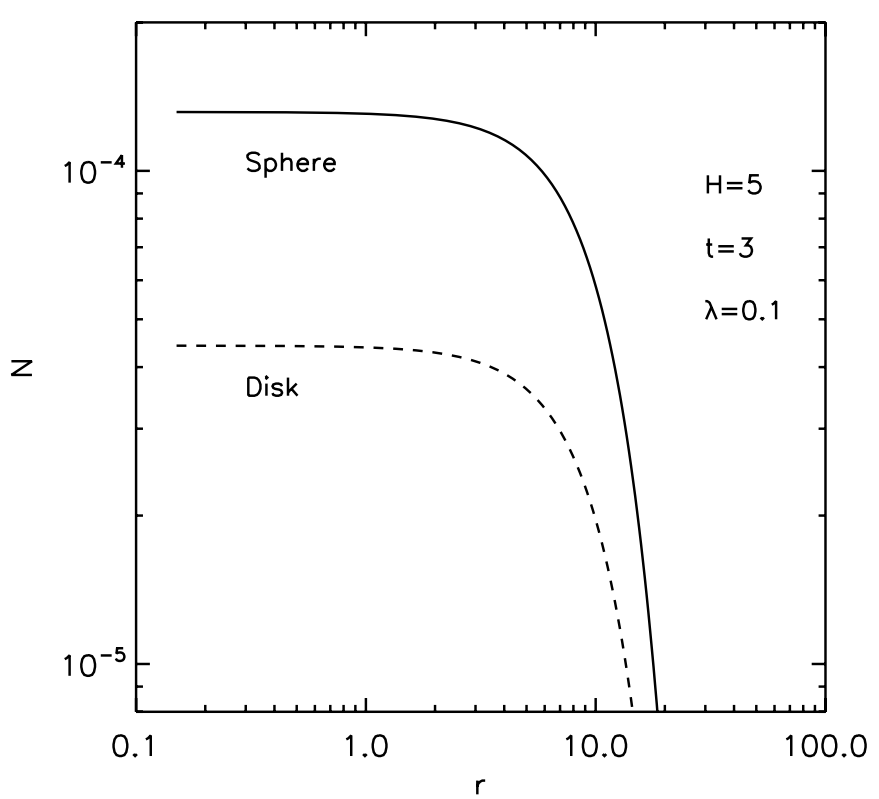

Figure 1. CR density as a function of $r(\rho)$ for isotropic diffusion (solid line) and disk geometry (dashed). The parameters are $t=3 \mathrm{Myr}, H=5 \mathrm{kpc}$, and $\lambda=0.1 \mathrm{kpc}$.

The anisotropy in the case of a single GRB is the same as in Equation (4), using $\rho$ in lieu of $r$. The ratio between the cosmicray density calculated for a disk geometry and that for spherical symmetry is

$$
R=\sqrt{2 \pi \frac{t}{\tau_{\mathrm{esc}}}} \exp \left(-\frac{t}{\tau_{\mathrm{esc}}}\right)
$$

which, as expected, becomes small if $t \gg \tau_{\text {esc }}$. A comparison of the radial dependence of the particle density for spherical symmetry as opposed to disk geometry is shown in Figure 1. To be noted is that the radial distribution is unchanged, the only difference is seen in the normalization.

\subsection{Discreteness Anisotropy}

The above discussion considered anisotropy from a single source in conditions of high intermittency. When many sources contribute, the anisotropy is reduced by a factor of order the square root of the number of contributing sources. We can formally define intermittency for the case of a disk geometry with absorbing boundaries as follows: the CR escape time from the disk, $\tau_{\text {esc }}$, if the CRs are produced in the Galactic plane, is $\tau_{\mathrm{esc}}=H^{2} /(2 D)=3 H^{2} /\left(2 \lambda_{\mathrm{mfp}} c\right)$. Here $D$, the CR diffusion coefficient, is written as $\lambda_{\text {mfp }} c / 3$, where $\lambda_{\text {mfp }}$ is the CR mean free path. The intermittency factor $I$ can be defined to be $1 / M_{\mathrm{CR}}$, where $M_{\mathrm{CR}}$ is the number of CR episodes per diffusion area over one escape time. Because the CR escape time is the time required to diffuse the height of the disk, $H$, the diffusion area over one escape time is $\pi H^{2}$, so $M_{\mathrm{CR}}=q \tau_{\mathrm{esc}} H^{2} / R^{2}$, where $R$ is the radius of the Galaxy and $q$ is the rate of CR production events in the entire Galaxy. (For SNe, $q_{\mathrm{SN}}$ is thought to be about $1 / 30$ $\mathrm{yr}$, and for GRB, $q_{\mathrm{GRB}}$ is thought to be somewhere between 30,000 years and 3 million years.) Sources much further away than $H$ are attenuated by escape and do not contribute significantly. Finally, we write $M_{\mathrm{CR}}$ as

$$
M_{\mathrm{CR}}=\frac{3 H^{4} q}{2 R^{2} \lambda_{\mathrm{mfp}} c}
$$

and, assuming that the average distance to the sources is of order $H$ and using Equation (3), we write the anisotropy in the case of $M$ contributing sources as

$$
\delta_{M} \approx \frac{\lambda_{\mathrm{mfp}}}{H \sqrt{M_{\mathrm{CR}}}}=\left(\frac{\lambda_{\mathrm{mfp}}}{H}\right)^{3 / 2} \frac{R}{H} \sqrt{\frac{2 c}{3 q H}} .
$$

The exact anisotropy of course depends on time and numerical factors of order unity. It may be increased by an inhomogeneous spatial distribution of CR sources in the Galaxy, and therefore in subsequent sections we shall consider the problem with Monte Carlo generation of CR events. It can be seen however that $\delta_{M}$, at EeV energies, can be considerable. For a Galactic magnetic field of $10 \mu \mathrm{G}$, the gyroradius of $3 \mathrm{EeV}$ protons is $0.3 \mathrm{kpc}$, and thus the factor $\left[\lambda_{\mathrm{mfp}} / H\right]^{3 / 2}$ is more than $1 \%$ even if $\lambda_{\mathrm{mfp}}$ is a single gyroradius. The factor $R / H$ is considerably larger than unity except for a rather thick disk, and $\sqrt{\frac{2 c}{3 q H}}$ is at least of order unity for GRBs, and about $10^{-1.5}$ for SNe. Unless $H / R$ is chosen to be of order unity, the diffusion is sub-Bohmian, or the trans-EeV CR are heavy nuclei, the anisotropy exceeds the experimental limit of $1 \%$ set by the AUGER experiment (Pierre AUGER Collaboration et al. 2011). The inverse cubic dependence of $\delta$ on $H$ constrains $H$ to be larger than $3 \mathrm{kpc}$ if $\mathrm{SNe}$ are the sources of UHE Galactic CR, and, if GRBs are the sources, appears to require them to be intermittent.

The qualitative point is that, if the sources are distributed in the Galactic plane, then "Olbers' paradox" does not apply and close sources contribute as much as distant ones. This allows the possibility of discrete anisotropy at ultra-high energies.

\subsection{Intermittency}

\subsubsection{Spectra}

Generally, GRBs in the Galaxy are expected every million years or so, the exact rate depending on the beaming fraction and the detailed scaling of long GRB with star formation and metallicity (for a detailed review, see Gehrels et al. 2009). Therefore, only a small number of GRBs can contribute to the particle flux at the solar circle, and their relative contribution depends on the location and explosion time of the GRB. Variations in the local particle flux must be expected, and neither the particle spectrum from an individual GRB nor the spectrum calculated for a homogeneous source distribution are good proxies. To fully account for discreteness of GRBs in space and time, we can use the method of Monte Carlo to randomly place GRBs in the Galaxy with given spatial probability distribution in galactocentric radius,

$$
P\left(r_{\mathrm{GC}}\right)=\frac{2 r_{\mathrm{GC}}}{r_{0}^{2}} \exp \left(-\frac{r_{\mathrm{GC}}^{2}}{r_{0}^{2}}\right)
$$

with scale $r_{0}=5 \mathrm{kpc}$, and with given GRB rate, e.g.,

$$
P(t)=0.5 \mathrm{Myr}^{-1} \text {. }
$$

The assumed galactocentric distribution is an approximation to the distributions of baryons and star formation in the Galaxy, which are reasonable proxies for the distribution of SNe. The distribution of long GRB may be skewed toward the outer Galaxy by metallicity-related selection effects (e.g., Levesque et al. 2010; Levesque 2011). In this paper, we keep fixed the galactocentric distribution of UHECR sources. In a forthcoming 


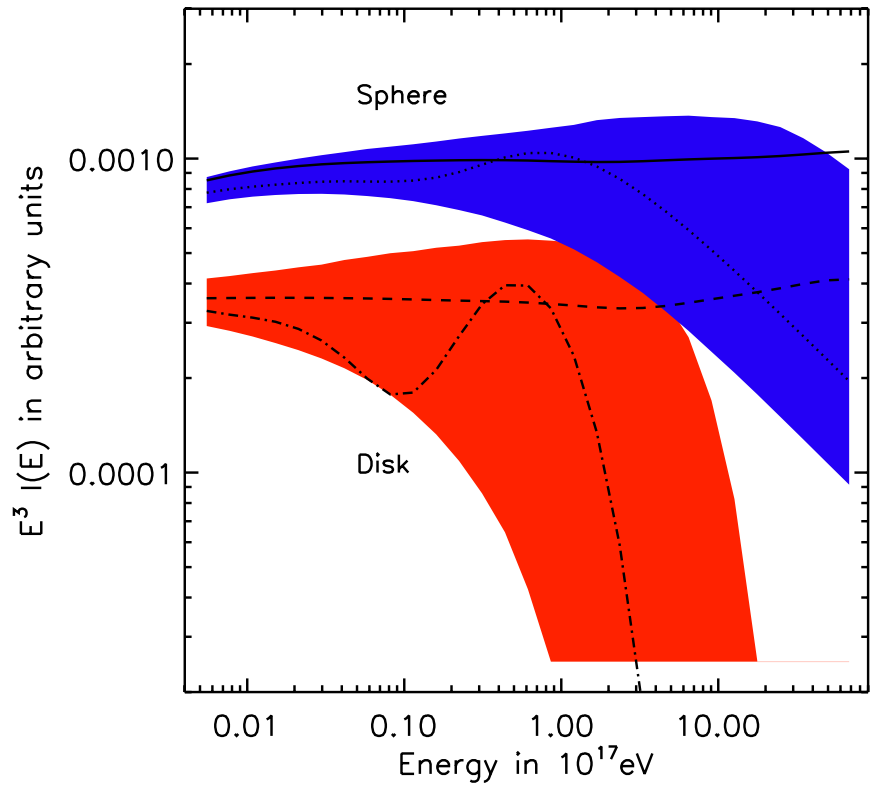

Figure 2. Proton spectra at the solar circle expected for a diffusion coefficient with Bohmian energy scaling (case A). For spherical geometry, the solid line shows the time-averaged particle spectrum, whereas the dotted line denotes one randomly selected case. The blue band indicates the central $68 \%$ containment region for the particle flux at the given energy. The dashed line, dash-dotted line, and the red band (partially obscured) are the same for disk geometry.

(A color version of this figure is available in the online journal.)

study, we shall explore the impact of sources in the outer Galaxy, e.g., short GRBs.

Each GRB is assumed to inject particles over $10^{4} \mathrm{yr}$ with the same total energy content and spectrum

$$
Q(E)=Q_{0} E^{-s}
$$

The actual choice of spectral index is irrelevant for the calculation, because it propagates right into the final expressions for the particle spectra (cf. Equations (2) and (18)). Likewise, it is sufficient to calculate the variations in the local flux for protons, because nuclei of charge $Z$ and energy $E_{Z}$ behave like a proton of energy $E=E_{Z} / Z$ (cf. Section 2.1.1). The halo size is again $H=5 \mathrm{kpc}$.

Below we show results for two different energy dependences of the diffusion coefficient, or mean free path

$$
\text { Case A: } \quad \lambda_{\text {mfp }}=10 r_{L}
$$

$$
\text { Case B: } \quad \lambda_{\operatorname{mfp}}=10 r_{L} E_{17}^{-0.5}
$$

where the Larmor radius is calculated for a proton in a $10 \mu \mathrm{G}$ magnetic field and $E_{17}=E /\left(10^{17} \mathrm{eV}\right)$. Case B thus uses a diffusion coefficient that increases with the square root of the energy, similar to that conventionally used for Galactic cosmic rays below the knee. Note that for case $A$ the diffusion approximation breaks down at approximately $10^{18} \mathrm{eV}\left(10^{19} \mathrm{eV}\right.$ for case B).

For both cases A and B we have calculated spectra for $10^{4}$ random sets of GRBs. As we demonstrated before, energy losses through inelastic collisions can be ignored. To reach a quasisteady state, we need to sample the contributions of all GRBs for a time period much longer than the escape time. We chose to integrate over $6 \mathrm{Gyr}$ in time, which is long enough at all energies and for all choices of $\lambda_{\text {mfp }}$ used in this study. Overall,

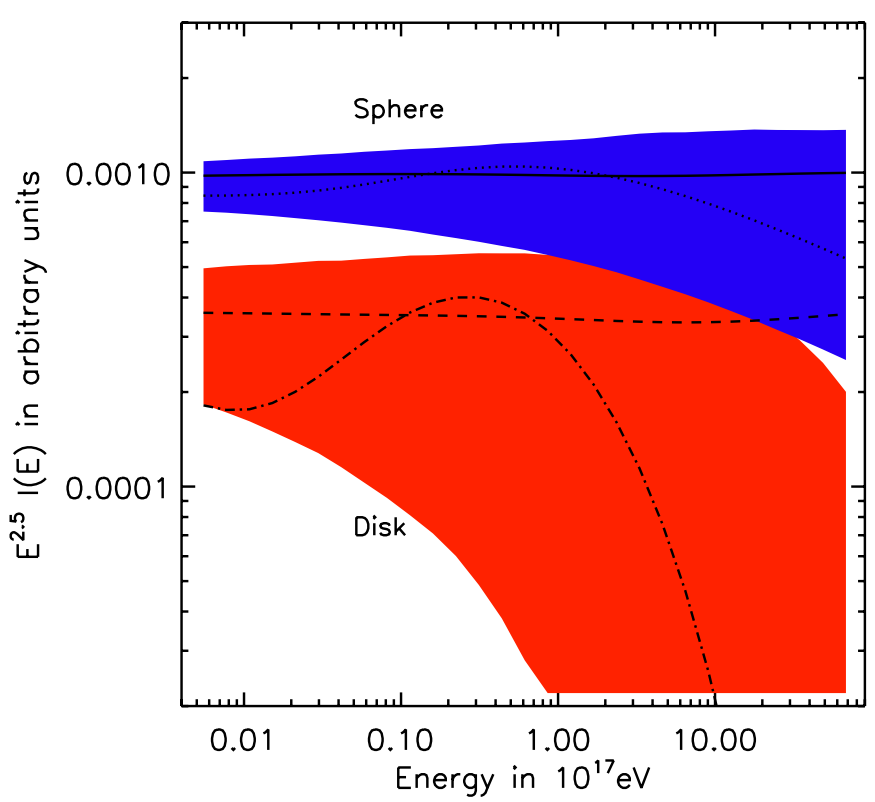

Figure 3. Proton spectra at the solar circle expected for a diffusion coefficient scaling with $\sqrt{E}$ (case B). For spherical geometry, the solid line shows the timeaveraged particle spectrum, whereas the dotted line is one randomly selected case. The blue band indicates the central $68 \%$ containment region for the particle flux at the given energy. The dashed line, dash-dotted line, and the red band (partially obscured) are the same for disk geometry. Note that the $y$-axis is now $E^{2.5} I$.

(A color version of this figure is available in the online journal.)

the method is the same as that used to model the transport of cosmic-ray electrons in the Galaxy (Pohl \& Esposito 1998; Pohl et al. 2003). Results are shown in Figure 2 for case A, and in Figure 3 for case B, both with injection index $s=2$. The shaded areas in the figures indicate the central $68 \%$ containment region for the particle flux, meaning $16 \%$ of the simulated spectra fall below the colored range, and another $16 \%$ are higher.

To be noted from the figures are

1. The energy dependence of the diffusion coefficient influences the particle spectrum observed at Earth. Features in the observed spectrum could thus arise from changes in the energy dependence, e.g., from shallow at lower energies to Bohmian at higher energies, without requiring any structure in the source spectrum (see also Calvez et al. 2010).

2. The solution for disk geometry is typically a factor 2-3 lower than that for spherical geometry, reflecting the fact that the free-escape boundary in the halo introduces particle losses.

3. Intermittency is strong for a GRB rate below 1 per Myr, in particular for the more realistic disk geometry. In essence, the local UHECR spectrum from Galactic GRBs is unpredictable if the scattering mean free path exceeds about $100 \mathrm{pc}$, which for the parameters used here is the case above $10^{17} \mathrm{eV}$ for protons, and above $3 \times 10^{18} \mathrm{eV}$ for iron. Model fits of single-source spectra can thus be very misleading (cf. Wick et al. 2004).

4. Particularly interesting is the finding that the time-averaged spectrum can be outside the $68 \%$ containment region, which arises from the very rare GRBs that are both recent and near. It indicates that it is unlikely that we observe the timeaveraged spectrum. The median of the distribution shows a spectral cut-off, which is therefore the most likely outcome, 


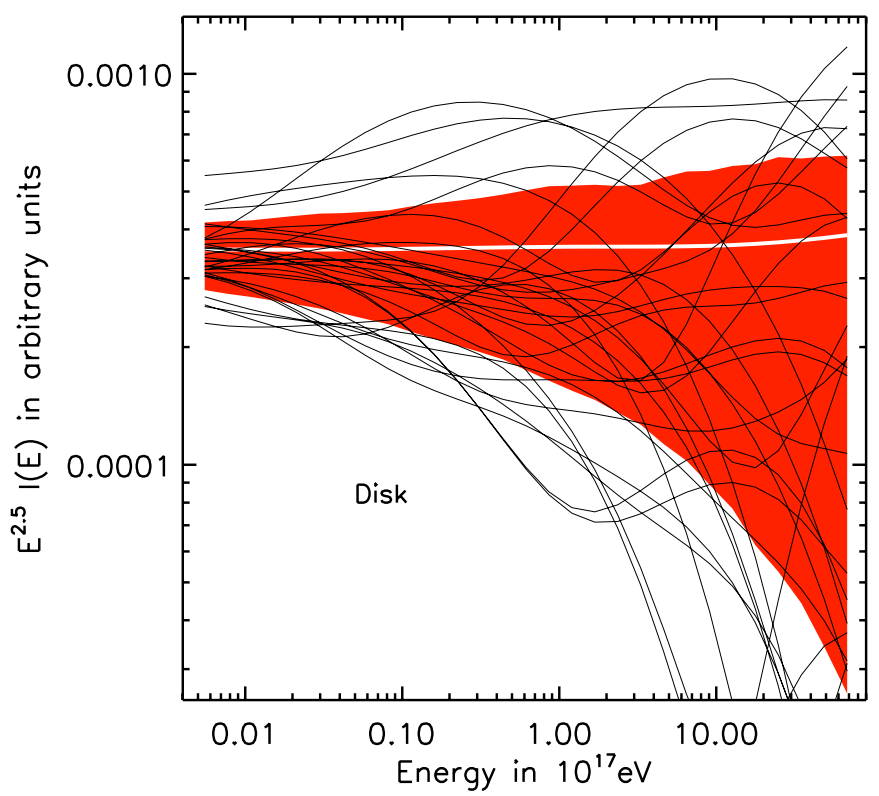

Figure 4. Proton spectra at the solar circle expected for a diffusion coefficient scaling with $\sqrt{E}$ (case B) in disk geometry. The parameters are the same as in Figure 3, except that we have increased the GRB rate to $5 \mathrm{Myr}^{-1}$. The thin solid lines show 31 randomly selected spectra, whereas the time average is now given by the thick white line.

(A color version of this figure is available in the online journal.)

whereas the time-averaged spectrum does not and thus is not useful in a comparison with data.

5. The actually expected spectra display bumps unrelated to both source and propagation physics, some of which may indeed be observed (Arteaga-Velázquez et al. 2010). The absence of very large bumps in the observed UHECR spectra suggests that either the mean free path for scattering is smaller than assumed here, or the rate of cosmic-rayproducing GRBs in the Galaxy exceeds 1 per Myr, at which the amplitude of such bumps becomes smaller (see Figure 4). Generally, careful accounting of the statistical fluctuations is mandatory for properly estimating the local UHECR spectrum from GRBs (cf. Calvez et al. 2010).

\subsubsection{Anisotropy}

If more than one GRB contributes to the local UHECR spectrum, their anisotropy signal will generally not line up and can thus not be simply averaged. If all sources reside in the Galactic plane, the entire problem is essentially twodimensional. We use an Eddington approximation for the UHECR intensity from a single GRB,

$$
I=I_{0}+I_{1} \cos \theta=I_{0}(1+\delta \cos \theta),
$$

where $\theta$ is the angle relative to the line of sight from the GRB to us. The GRB is located at Galactocentric coordinates $\left(r_{\mathrm{GC}}, \phi\right)$, which translates to distance, $r$, and line-of-sight direction relative to the anticenter, $\psi$, as

$$
r^{2}=64+r_{\mathrm{GC}}^{2}-16 r_{\mathrm{GC}} \cos \phi
$$

and

$$
\cos \psi=\frac{8-r_{\mathrm{GC}} \cos \phi}{r} \quad \sin \psi=\frac{r_{\mathrm{GC}} \sin \phi}{r},
$$

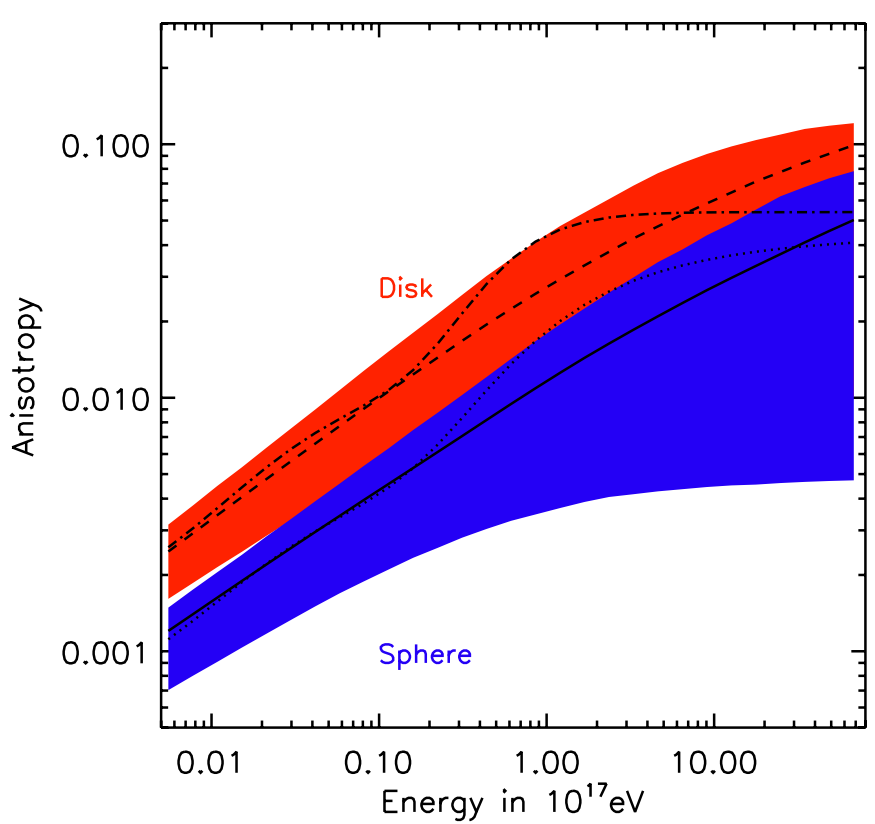

Figure 5. Expected anisotropy for the same parameters as in Figure 3. For spherical geometry, the solid line shows the average anisotropy, whereas the dotted line is one randomly selected case. The blue band indicates the $68 \%$ containment region for the anisotropy at the given energy. The dashed line, dash-dotted line, and the red band (partially obscured) are the same for a disk geometry.

(A color version of this figure is available in the online journal.)

where the distance unit kpc is used. The local anisotropy can be expressed through projection in the anticenter direction, $\vec{e}_{x}$, and in a direction perpendicular to it, $\vec{e}_{y}$,

$$
I_{x}=\delta I_{0} \cos \psi \quad I_{y}=\delta I_{0} \sin \psi .
$$

After summing over all sources,

$$
I_{\mathrm{tot}}=\sum I_{0} \quad I_{\mathrm{tot}, x}=\sum I_{x} \quad I_{\mathrm{tot}, y}=\sum I_{y}
$$

the observed anisotropy is

$$
\delta_{\mathrm{tot}}=\frac{\sqrt{I_{\mathrm{tot}, x}^{2}+I_{\mathrm{tot}, y}^{2}}}{I_{\mathrm{tot}}}
$$

and the direction is given by

$$
\alpha=\arctan \left(\frac{I_{\mathrm{tot}, y}}{I_{\mathrm{tot}, x}}\right) .
$$

Figure 5 shows the anisotropy signal for the parameters of case B (see Equation (26)). For Bohm-like scaling of the mean free path (case A), the anisotropy is the same at $10^{17} \mathrm{eV}$ but has a steeper energy dependence. The current experimental limits on $\delta_{\text {tot }}$ set by the Auger Observatory are $\delta_{\text {tot }} \leqslant 0.01$ in the $[0.4,4] \mathrm{EeV}$ range and $\delta_{\text {tot }} \leqslant 0.05$ in the $[4,40] \mathrm{EeV}$ range (Armengaud 2008; Blümer et al. 2009; Bonino et al. 2009; Pierre AUGER Collaboration et al. 2011). To be noted from the figure is that the anisotropy for protons above $10^{17} \mathrm{eV}$ is high compared with the Auger limits, in particular for the more realistic disk geometry. Note that the average anisotropy (dashed and solid lines) is independent of the GRB rate. A substantial contribution of heavy nuclei, a small mean free path, or extragalactic UHECR would be needed to satisfy the anisotropy limit. Figure 6 displays 


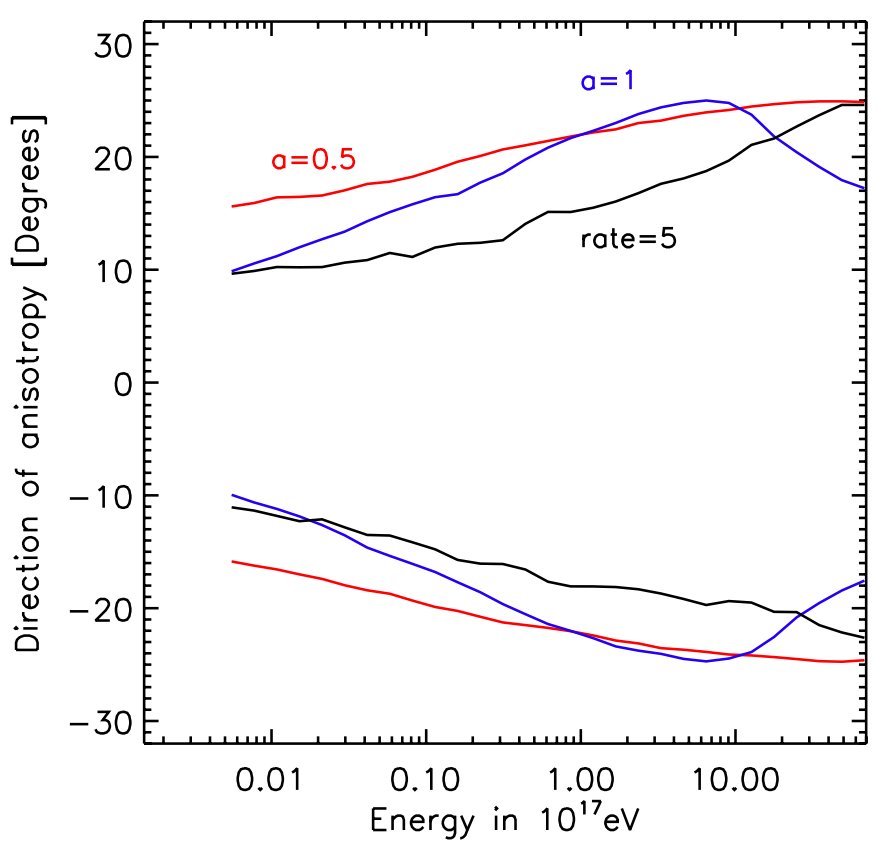

Figure 6. Distribution of anisotropy directions relative to the anticenter for disk geometry. The red and blue lines mark the boundaries of the $68 \%$ containment range for shallow $(a=0.5)$ and Bohmian $(a=1)$ energy scaling of the mean free path, respectively, both for a low GRB rate of $0.5 \mathrm{Myr}^{-1}$. For comparison, the black line is for $a=0.5$, but high GRB rate $5 \mathrm{Myr}^{-1}$.

(A color version of this figure is available in the online journal.)

the variation in the anisotropy direction, suggesting that one may underestimate the anisotropy if one only considers its projection on the anticenter direction, fairly independent of the choice of parameters.

\section{COMBINED ANALYSIS}

We now try to construct a model that reproduces the spectrum of cosmic rays between $4 \times 10^{16} \mathrm{eV}$ and $4 \times 10^{18} \mathrm{eV}$ together with the anisotropy limits and the composition. We use data of the Kascade-Grande Collaboration (Apel et al. 2009; ArteagaVelázquez et al. 2010), ranging from $2 \mathrm{PeV}$ to $0.5 \mathrm{EeV}$, HiRes monocular data between 0.2 EeV and $3 \mathrm{EeV}$ (Abbasi et al. 2005), and the Auger Collaboration (Pierre AUGER Collaboration et al. 2010) above $1 \mathrm{EeV}$.

At $10^{18} \mathrm{eV}$ the anisotropy is low, $\delta \lesssim 0.01$ (the $99 \%$ upper limit is 0.014; Pierre AUGER Collaboration et al. 2011), and the composition is light, though not necessarily dominated by protons (Abraham et al. 2010). Figure 5 suggests that the mean free path must be relatively small to accommodate the anisotropy limits for light nuclei such as helium. Cosmic-ray propagation models can reproduce the composition and particle spectra in the GeV-TeV band for a shallow energy dependence of the diffusion coefficient $\left(D \propto E^{0.33}\right)$, if stochastic reacceleration is included (Strong \& Moskalenko 1998; Maurin et al. 2001; Jones et al. 2001). If we were to extrapolate their results, the proton mean free path at $10^{17} \mathrm{eV}$ would be $\sim 500 \mathrm{pc}$, i.e., larger than what we have assumed above. Obviously, we cannot permit the extrapolation to hold, if Galactic GRBs are to account for the observed UHECRs in the $10^{18} \mathrm{eV}$ energy band. The mean free path must be smaller than the extrapolation, but how much smaller, in particular how small compared with the particle Larmor radius?

Figure 7 shows spectra for a possible model configuration, where for simplicity we display only spectra for protons, helium,

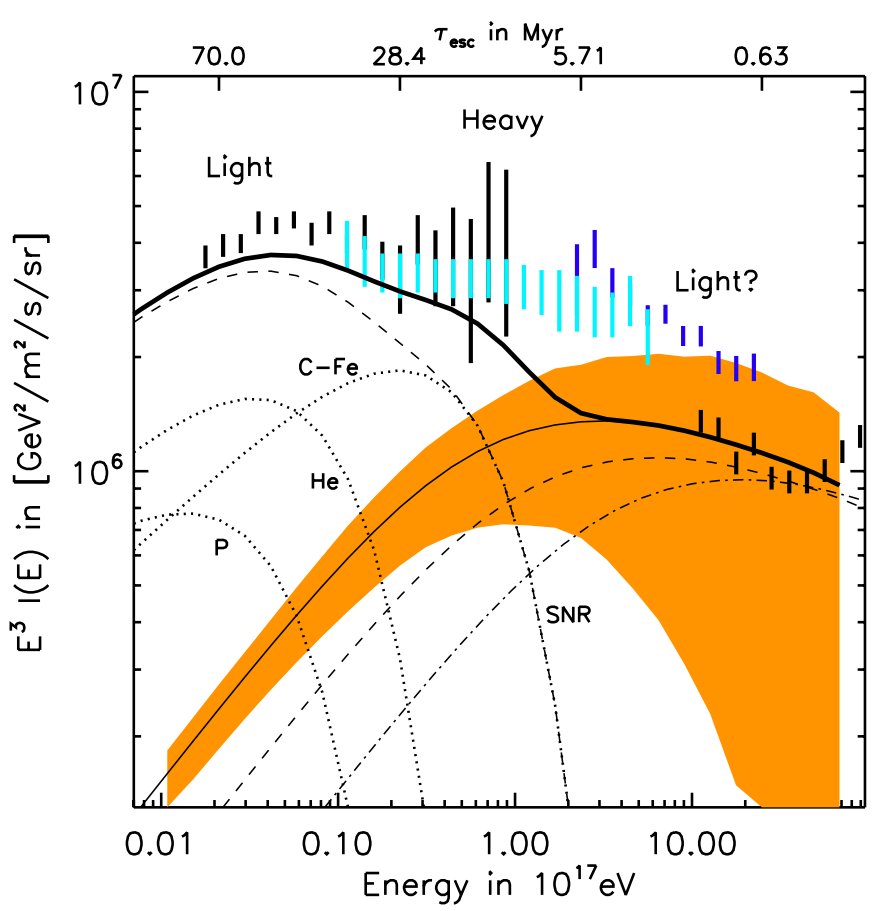

Figure 7. Example of model spectra for cosmic-ray protons, helium, and carbon nuclei, including the $68 \%$ variation range in the case of protons. The solid line denotes the time-averaged spectrum of protons, the dashed line displays the same for helium, and the dash-dotted line is for carbon. The mean free path follows Equation (34), and the corresponding escape timescales are indicated at the top axis. Also shown are the spectra measured with Kascade-Grande below $0.5 \mathrm{EeV}$, Auger above $1 \mathrm{EeV}$, and HiRes in between, together with labels indicating the composition. The offset between spectra from different experiments is likely due to errors in the absolute energy scale. For comparison, we also display correspondingly labeled simple mock spectra of protons, helium, and $\mathrm{C}-\mathrm{Fe}$ group particles possibly produced in SNR. The thick triple-dot-dashed line indicates the total of protons from GRB and all particles from SNR.

(A color version of this figure is available in the online journal.)

and carbon as proxies for light and heavy nuclei, respectively. The GRB rate is set to $P(t)=1 \mathrm{Myr}^{-1}$ and the source spectral index is $s=2.1$. The mean free path transitions from a shallow energy dependence to Bohmian scaling $(\propto E)$ as the particle energy increases,

$$
\lambda_{\mathrm{mfp}}=\lambda_{0} E^{0.3}\left[1+\frac{E}{60 \mathrm{PeV}}\right]^{0.7},
$$

where $\lambda_{0}$ is chosen so a proton has a mean free path that is a certain multiple (unity in Figure 7) of $11 \mathrm{pc}$ at $10^{17}$ $\mathrm{eV}$, its Larmor radius in a $10 \mu \mathrm{G}$ magnetic field. The top axis of Figure 7 indicates the escape time at a few energies. The spectrum below $10^{17} \mathrm{eV}$ is far below observed values to accommodate other Galactic sources of cosmic rays, such as SNR or pulsar wind nebulae, for which we plot simple power laws with exponential cut-off at $Z \cdot(3 \mathrm{PeV})$ to guide the eye, based on Ahn et al. (2010). For that we assume the averages $\langle A\rangle=25$ and $\langle Z\rangle=12.5$ for the $\mathrm{C}-\mathrm{Fe}$ group and the following power-law indices: $s=2.66$ for protons, $s=2.58$ for helium, and $s=2.56$ for $\mathrm{C}-\mathrm{Fe}$. It is apparent that the energy scales of HiRes and Auger are discrepant. To fully fit the data we need to increase the normalization of the GRB-particle spectrum and either disregard the Auger data or, alternatively, distrust HiRes and require a fluctuation in the local spectrum that makes the GRB-produced protons somewhat softer than the average spectrum plotted in Figure 7. Such a deviation would 


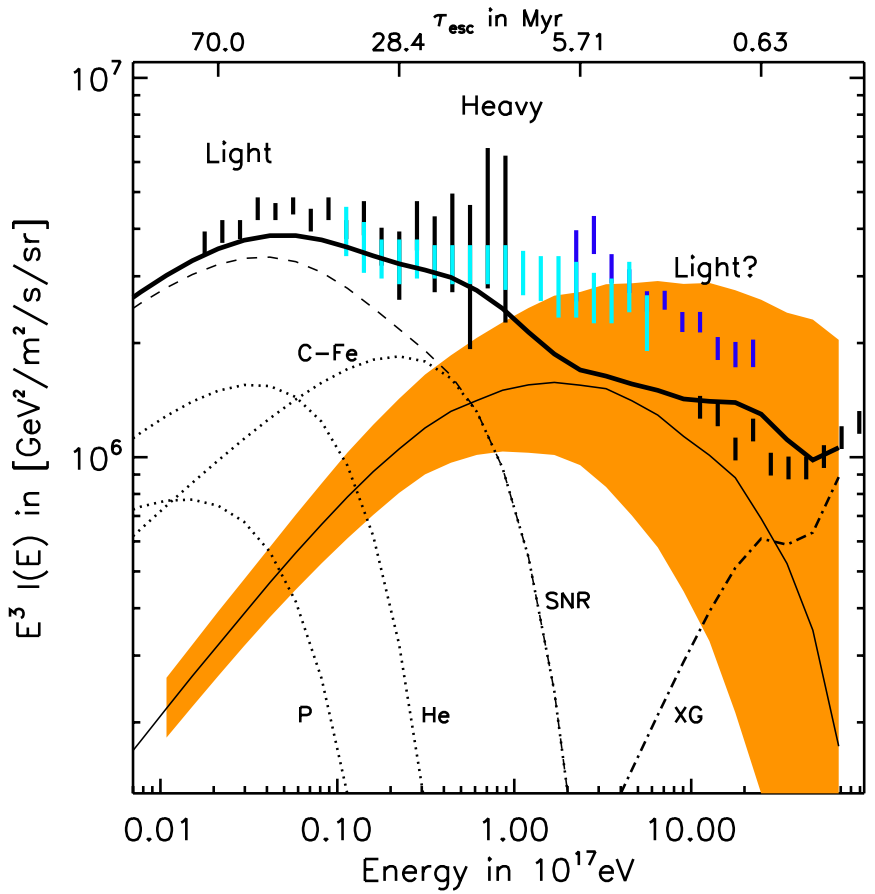

Figure 8. Example of model spectra for cosmic-ray protons in the presence of an extragalactic proton component. The solid line denotes the median spectrum of protons, and the dash-dotted line displays the extragalactic proton spectrum. Otherwise, everything is the same as in Figure 7.

(A color version of this figure is available in the online journal.)

be common, because the fluctuation amplitude at escape times below 1 Myr is large for the GRB rate used here, one per Galaxy per Myr.

The required spectral shape above $1 \mathrm{EeV}$ depends on the properties of the extragalactic component that dominates above the ankle. Berezinsky et al. (2006) have demonstrated that the effect of the pair-production threshold on an extragalactic component composed solely of protons can match the ankle fairly well. In Figure 8, we add such a component using a simple approximation of the pair-production loss rate $\left(E \propto E \Theta\left(E-E_{\mathrm{thr}}\right)\right.$, assuming no evolution, i.e., $m=0$ in Berezinsky et al. (2006), and integrating out to a redshift $z_{\max }=5$ ). The evolution and redshift cut-off can in principle be constrained using the neutrino and gamma-ray backgrounds (e.g., Wang et al. 2011), but such an endeavor is beyond the scope of this paper. In calculating the anisotropy (cf. Figure 9), we assume the extragalactic component to be perfectly isotropic. The motion of the Sun and the Galaxy relative to the CMB frame suggests the presence of a Compton-Getting anisotropy of amplitude $\sim 0.5 \%$ (Kachelrieß $\&$ Serpico 2006), but it is unclear to what degree scattering in the Galaxy can reduce the observed anisotropy. Assuming no anisotropy in the extragalactic component therefore constitutes a best-case scenario (in the sense of diluting the anisotropy) that is likely too optimistic. Note that we slightly rescaled the component from galactic GRB and plot the median instead of the time average.

The average spectrum mainly depends on the absolute value of the mean free path at high energies. We can calculate the cosmic-ray source power required to sustain the observed flux of UHECRs at $10^{18} \mathrm{eV}$ as a function of the mean free path. For the parameters used here, and an injection spectrum $\propto E^{-2.1}$ extending from the $\mathrm{GeV}$ band to the highest energies, fitting the

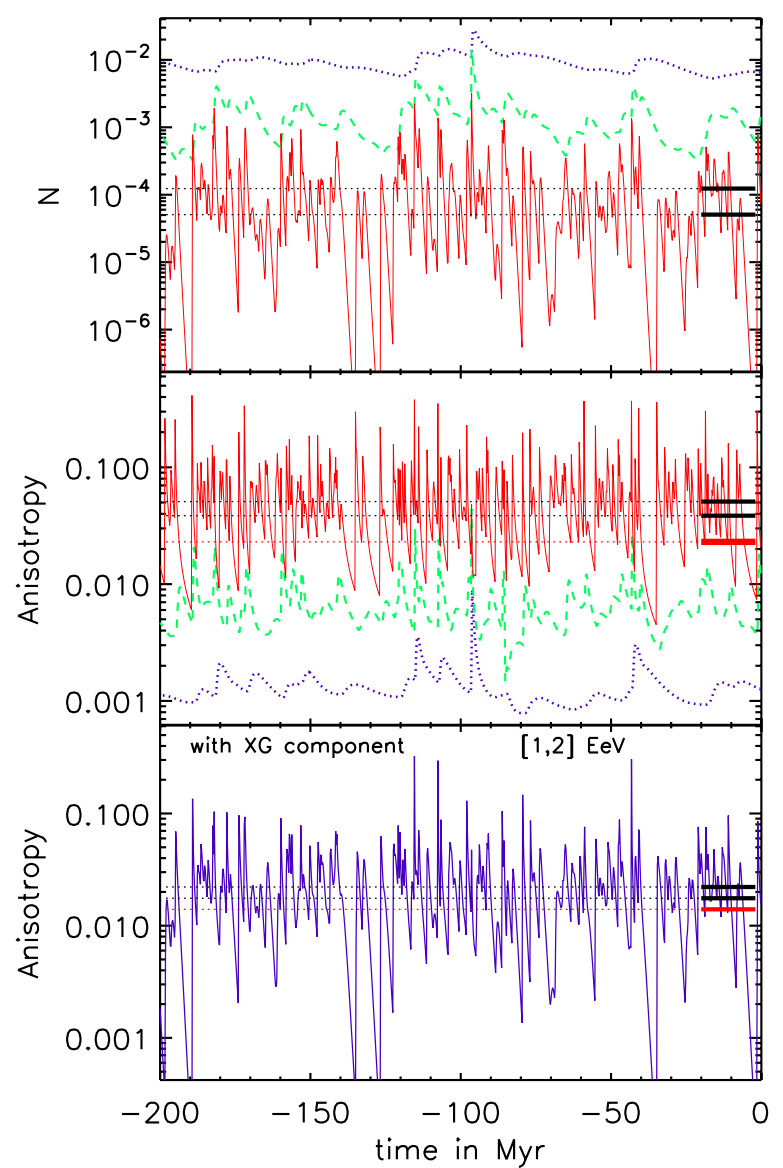

Figure 9. Top panel: 200 Myr light curve of the local proton flux (cf. Figure 7) in arbitrary units at three different escape times relative to the inverse source rate of $1 \mathrm{Myr}$ : the dotted (blue) line is for $\tau_{\mathrm{esc}}=20 \mathrm{Myr}$, the dashed (cyan) line for $\tau_{\text {esc }}=4.5 \mathrm{Myr}$, and the solid (red) line for $\tau_{\mathrm{esc}}=0.5 \mathrm{Myr}$. The two black bars on the right of the panels, extended as thin dashed lines, indicate the median and the average of the light curves at $\tau_{\mathrm{esc}}=5 \times 10^{5} \mathrm{yr}$, where the lower bar always indicates the median. Middle panel: temporal variation of the anisotropy at the same escape times. For the diffusion parameters chosen in the text, where the mean free path is chosen conservatively as the gyroradius for the two highest energies, the dotted (blue) line is for $0.024 \mathrm{EeV}$, the dashed (cyan) line for $0.24 \mathrm{EeV}$, and the solid (red) line for $2.4 \mathrm{EeV}$. The red bar on the right indicates the $99 \%$ upper limit set by Auger. Bottom panel: temporal variation of the anisotropy for the model including an extragalactic proton component (cf. Figure 8), here only between $1 \mathrm{EeV}$ and $2 \mathrm{EeV}$.

(A color version of this figure is available in the online journal.)

observed flux of UHECRs at $10^{18} \mathrm{eV}$ requires the source power

$$
P_{\mathrm{CR}}=P(t) \int_{1 \mathrm{GeV}} d E E Q_{0} E^{-s} \simeq \frac{\lambda_{\mathrm{mfp}}}{r_{L}}\left(10^{37} \mathrm{erg} \mathrm{s}^{-1}\right) .
$$

The source power in the energy interval $\left[10^{17}, 10^{18}\right] \mathrm{eV}$ alone is

$$
P_{\mathrm{EeV}} \simeq \frac{\lambda_{\mathrm{mfp}}}{r_{L}}\left(3.5 \times 10^{35} \mathrm{erg} \mathrm{s}^{-1}\right),
$$

which is about $10^{-5}$ of the total CR luminosity of the Galaxy. By way of comparison, the source intensity of alternative sources to SNR, if they are to supply all Galactic CR at energies above the Lagage-Cesarsky limit of $E_{\mathrm{LC}}=10^{14.5} \mathrm{eV}$ for SNR, must be the Galactic luminosity of CR above these energies, i.e.,

$$
\begin{aligned}
P_{\mathrm{SNR}} & \approx 10^{(-0.7)(5.5)} L_{\mathrm{GeV}} \frac{\tau_{\mathrm{esc}}(\mathrm{GeV})}{\tau_{\mathrm{esc}}\left(10^{5.5} \mathrm{GeV}\right)} \\
& \simeq\left(10^{36.5} \mathrm{erg} \mathrm{s}^{-1}\right) \frac{\tau_{\mathrm{esc}}(\mathrm{GeV})}{\tau_{\mathrm{esc}}\left(10^{5.5} \mathrm{GeV}\right)} .
\end{aligned}
$$


Assuming that $\tau_{\text {esc }}(\mathrm{GeV}) / \tau_{\mathrm{esc}}\left(10^{5.5} \mathrm{GeV}\right) \geqslant 30$, this is about $10^{38} \mathrm{erg} \mathrm{s}^{-1}$ above $10^{5.5} \mathrm{GeV}$. On the other hand, SNe may supply the observed CR all the way to $10^{16} \mathrm{eV}$ if those at the highest energies are heavy nuclei.

What Figures 7 and 8 serve to demonstrate is that a GRBproduced cosmic-ray component can fill the gap between the contributions of ordinary galactic cosmic-ray sources and the super-ankle region likely supplied by extragalactic sources. The discreteness fluctuations are typically larger than experimental uncertainties. But what of the anisotropy and its variation?

The light curve shown in the top panel of Figure 9 shows that besides the increase in fluctuation amplitude, the fluctuation timescale decreases with particle energy (or more precisely escape probability). For the parameters used here, the escape time at $2.4 \mathrm{EeV}$ is about $5 \times 10^{5} \mathrm{yr}$. The flux variations can be compared with the temporal behavior of the anisotropy, shown in the middle panel of Figure 9. Already at $0.24 \mathrm{EeV}$ (the dashed, cyan line) the anisotropy hovers around $1 \%$. A clear correlation between flux and anisotropy is not discernible at higher energies, although at $0.024 \mathrm{EeV}$ some structures in flux and anisotropy coincide. However, other spikes in flux or anisotropy observed at $0.024 \mathrm{EeV}$ have no counterpart at higher energies, and vice versa. To be noted from the figures is that significant excursions to low anisotropy are rare. The minimum anisotropy is 0.0045 , whereas the minimum flux is $8 \times 10^{-9}$.

Figure 9 also indicates the median and the time average of the flux and anisotropy at $2.4 \mathrm{EeV}$. The average is what we would expect to observe if the number of UHECR sources were much larger. In contrast, the median is a typical result in the case of intermittent sources. To be noted from the figure is that both the average and the median at $2.4 \mathrm{EeV}$ are considerably in excess of the Auger limits. Nevertheless, the anisotropy falls below the $99 \%$ upper limit of $2.3 \%$ (for the energy band between 2 and $4 \mathrm{EeV}$; Pierre AUGER Collaboration et al. 2011) during about $25 \%$ of the time. The bottom panel of Figure 9 shows the anisotropy variation in the energy band $[1,2] \mathrm{EeV}$ for the case including an extragalactic proton component as in Figure 8, which we assume to be perfectly isotropic. The average anisotropy is slightly reduced, but we note that the anisotropy becomes very small during lulls, essentially because during these times the extragalactic component dominates the spectrum. Still, the anisotropy falls below the $99 \%$ upper limit of $1.4 \%$ only during $40 \%$ of the time.

During the long lulls the intensity dips as low as three or four orders of magnitude below the time average. This possibility would suggest an anthropic scenario whereby we live in highly unusual times, and that the present cosmic-ray intensity is, perhaps, far below usual conditions, which for some (possibly obscure) reason would be hostile to intelligent life and/or advanced civilization. Note that the extreme versions of this scenario, in which the present UHECR luminosity of the Galaxy is $\sim 10^{-4}$ below the average (and possibly an even larger factor below the most recent maximum), would require an average (or peak) luminosity above $100 \mathrm{GeV}$ or so that compares with (or exceeds) the present-day value, and hence an extremely flat Galactic source spectrum. This could possibly be tested or constrained by abundance measurements of terrestrial cosmogenic nuclei (TCNs). It should be remembered that the hypothesis of sharp time dependence applies only to the CR that escape rapidly enough, and the anisotropy bounds motivate this only at the highest energies. Thus, the TCN production rate as a whole is not necessarily strongly affected by the hypothesis,

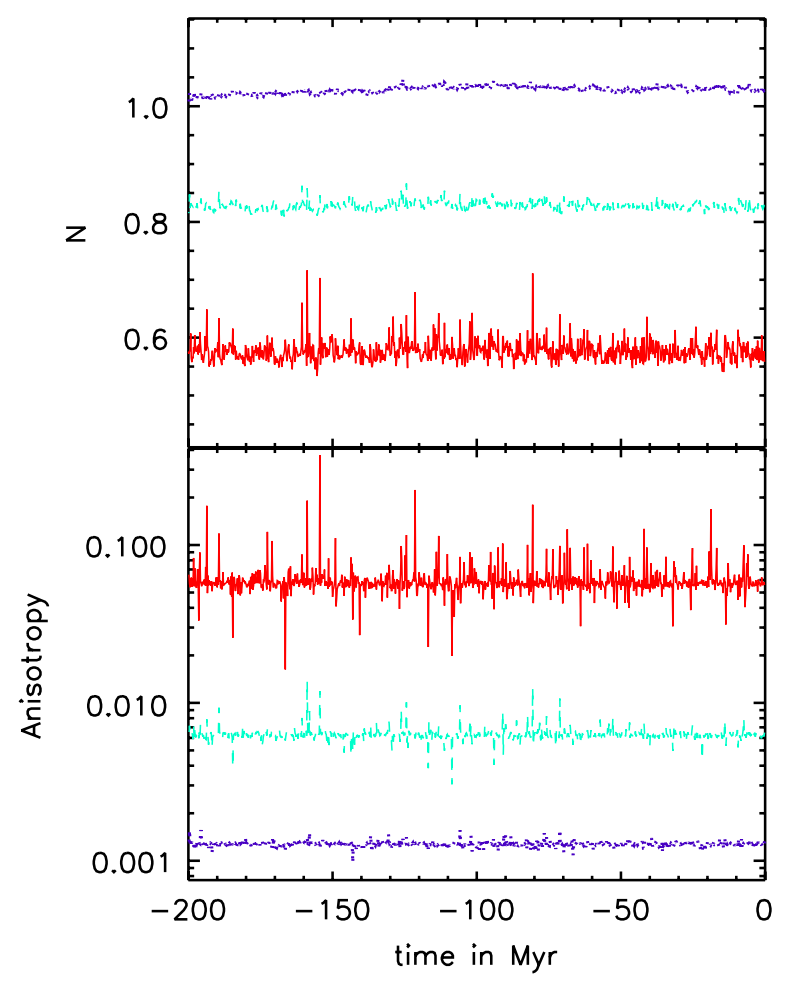

Figure 10. Top panel: $200 \mathrm{Myr}$ light curve of the local proton flux at the three different escape times denoted in Figure 9, assuming the protons originate in SNR with the parameters described in the text. Bottom panel: temporal variation of the anisotropy at the same energies.

(A color version of this figure is available in the online journal.)

so TCN anomalies are not firmly predicted by it. On the other hand, the production of ionizing particles at sea level per unit primary energy is an increasing function of the latter (assuming shower maximum occurs above sea level). Thus, for a perfectly flat spectrum (equal energy flux per decade of primary energy), most of the ionizing secondary flux at sea level, which might possibly be of astrobiological relevance, would come from CR at ultra-high energies. Further discussion of an anthropic scenario is beyond the scope of this paper, but will be the subject of future work. It is mentioned here to justify consideration of a scenario that would otherwise seem a priori improbable, but accounts for the remarkably low limits on anisotropy at ultra-high energy.

Also to be noted is that the high anisotropy is unavoidable in scenarios involving UHECR acceleration in SNRs, because intermittency is not an issue and there is little uncertainty concerning the spatial distribution of SNRs in the Galaxy. Light curves for an SNR origin are shown in Figure 10, for which we assumed an SN rate of one per 50 years. The rare spikes in intensity are caused by very nearby $\mathrm{SNe}$, for which their spatial and temporal evolution becomes an issue. Since we maintain the duration of the particle injection per source, $10^{-2} \mathrm{Myr}$, the spikes are likely clipped by temporal smoothing.

The anisotropy can in principle be reduced by choosing a smaller mean free path. Figure 11 shows the anisotropy for protons and a mean free path following Equation (34), but with $\lambda_{0}$ chosen so that at high energy $\lambda_{\mathrm{mfp}}=0.2 r_{L}$. The Auger limits, $\delta \lesssim 0.01$ at $1 \mathrm{EeV}$, can be satisfied with protons. The margin is not large, though, and a few more years of Auger operation should permit detection of an anisotropy signal, even for an unusually small mean free path. 


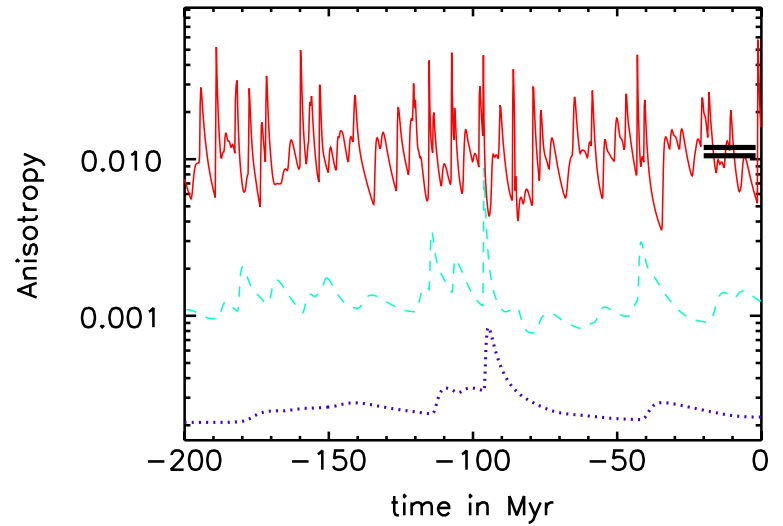

Figure 11. Temporal variation of the anisotropy as in the bottom panel of Figure 9, but for a smaller mean free path a factor five smaller than assumed there, i.e., $\lambda_{\mathrm{mfp}}=0.2 r_{L}$ at high energies. Again the average and the median at $2.4 \mathrm{EeV}$ are indicated and are both close to $1 \%$, approximately at the level of the Auger upper limits.

(A color version of this figure is available in the online journal.)

\section{SUMMARY AND DISCUSSION}

We have calculated the time-dependent transport of UHECRs in the Galaxy, assuming it can be described as isotropic diffusion. We have in particular investigated the question, whether or not Galactic long GRB can provide the observed particles up to the ankle, which is a natural transition point to extragalactic particles. In an earlier paper of the same series (Paper II) it was demonstrated that if extragalactic GRBs are to produce UHECRs above the ankle, Galactic GR should contribute a large flux of sub-ankle particles. Our present findings can be summarized as follows.

1. With the assumption of spherical symmetry, one overestimates the UHECR flux by a factor of a few and likewise underestimates the anisotropy.

2. Intermittency becomes serious if the mean free path for scattering exceeds $100 \mathrm{pc}$, unless the source rate is much higher than 1 per Myr. On average, Galactic long GRBs need to contribute only about $10^{37} \mathrm{erg} \mathrm{s}^{-1}$ in accelerated particles to fully account for the observed particle flux at $10^{18} \mathrm{eV}$, assuming a Bohmian mean free path at this energy.

3. UHECRs from Galactic long GRB can meet the observational limits on anisotropy only if the mean free path for scattering is sufficiently small. Contributing the observed sub-ankle particles (at $\left.10^{18} \mathrm{eV}\right)$ requires Bohmian diffusion if the UHECRs are as heavy as carbon. A light composition such as protons or helium requires sub-Bohmian diffusion, which is a highly unlikely situation for isotropic diffusion. We have not investigated the effects of a Galactic guiding field that may modify the probability of escape from the Galactic disk.

4. Auger data suggest that at $10^{18} \mathrm{eV}$ the composition is indeed light, thus posing a problem for the notion that Galactic GRBs (or any other source class with similar population statistic) produce the observed UHECRs up to the ankle. (This measurement is not undisputed, though, for the Kascade-Grande Collaboration has just published their analysis results which seem to favor a relatively heavy composition up to nearly $10^{18} \mathrm{eV}$; Arteaga-Velázquez et al. 2010.)

5. The UHECR composition is a very critical constraint, but its measurement is subject to considerable systematic uncertainties arising from its dependence on models for the development of air showers. It is imperative that measures be taken to better understand the air-shower physics near $10^{18} \mathrm{eV}$.

6. Much of the UHECR anisotropy arises from the expected location of long GRBs in the inner Galaxy. Observations of GRB host galaxies suggest that regions of low metallicity and high star formation may be the preferred sites of long GRBs (Levesque et al. 2010; Levesque 2011), which may skew the galactocentric distribution of long GRB toward the outer Galaxy. As there is no power problem with Galactic GRBs, it may be worthwhile to also consider short GRBs. They provide supposedly less power as a population, but they may have a very extended spatial distribution in the Galaxy, thus reducing the anisotropy (Berger 2010). The anisotropy arising from intermittency would remain in both cases and is the subject of a forthcoming publication.

7. Our study can be applied with little change to the case of an UHECR origin in SNRs, assuming very efficient magneticfield amplification can increase their ability to accelerate particles to energies significantly higher than $1 \mathrm{PeV}$ (e.g., Bell \& Lucek 2001). The spatial distribution in the Galaxy of long GRBs and SNRs can be expected to be similar, and therefore the average anisotropy is the same for both long GRBs and SNRs. We have verified that intermittency would be negligible in case of an SNR origin (unless of course the UHECRs come only from a rare subclass of $\mathrm{SNe}$ ) and therefore problems in simultaneously matching the composition and the anisotropy limits of UHECRs are inescapable for SNRs.

Support from the Israel Science Foundation, the Israel-U.S. Binational Science Foundation, and the Joan and Robert Arnow Chair of Theoretical Astrophysics is gratefully acknowledged.

\section{REFERENCES}

Abbasi, R., Abuzayyad, T., Amman, J., et al. 2005, Phys. Lett. B, 619, 271 Abraham, J., Abreu, P., Aglietta, M., et al. 2008, Phys. Rev. Lett., 101, 061101 Abraham, J., Abreu, P., Aglietta, M., et al. 2010, Phys. Rev. Lett., 104, 091101 Ahn, H. S., Allison, P., Bagliesi, M. G., et al. 2010, ApJ, 714, L89 Aloisio, R., Berezinsky, V., \& Gazizov, A. 2009, ApJ, 693, 1275

Apel, W. D., Arteaga, J. C., Badea, A. F., et al. 2009, Astropart. Phys., 31, 86 Armengaud, E. 2008, in Proc. 30th Int. Cosmic Ray Conf. (Mexico City), ed. R. Caballero et al., Vol. 4, 175-8

Arteaga-Velázquez, J. C., Apel, W. D., Bekk, K., et al. 2010, in Invited Talk at XVI: Int. Symp. on Very High Energy Cosmic Ray Interactions (arXiv:1009.4716)

Bell, A. R., \& Lucek, S. G. 2001, MNRAS, 321, 433

Berezinsky, V. S., Gazizov, A., \& Grigorieva, S. 2006, Phys. Rev. D, 74, 043005 Berezinskii, V. S., \& Grigoreva, S. I. 1988, Sov. Astron. Lett., 14, 1

Berger, E. 2010, ApJ, 722, 1946

Blümer, J., Engel, R., \& Hörandel, J. R. 2009, Prog. Part. Nucl. Phys., 63, 293

Bonino, R., et al. (for the Pierre Auger Collaboration) 2009, in Proc. 31st ICRC, Łodź, 24-7 (arXiv:0906.2347)

Büsching, I., Kopp, A., Pohl, M., et al. 2005, ApJ, 619, 314

Calvez, A., Kusenko, A., \& Nagataki, S. 2010, Phys. Rev. Lett., 105, 091101

Eichler, D., Guetta, D., \& Pohl, M. 2010, ApJ, 722, 543

Eichler, D., \& Pohl, M. 2011, ApJ, 738, L21 (Paper II)

Gehrels, N., Ramirez-Ruiz, E., \& Fox, D. B. 2009, ARA\&A, 47, 567

Ginzburg, V. L., \& Syrovatskii, S. I. 1964, The Origin of Cosmic Rays (New York: Macmillan)

Han, J. 2009, in IAU Symp. 259, Cosmic Magnetic Fields: From Planets to Stars and Galaxies, ed. K. G. Strassmeier, A. G. Kosovichev, \& J. E. Beckman (Cambridge: Cambridge Univ. Press), 455

Huang, C.-Y., Park, S.-E., Pohl, M., \& Daniels, C. D. 2007, Astropart. Phys., 27,429

Jones, F. C., Lukasiak, A., Ptuskin, V., \& Webber, W. 2001, ApJ, 547, 264 
Kachelrieß, M., \& Serpico, P. D. 2006, Phys. Lett. B, 640, 225

Kardashev, N. S. 1962, SvA, 6, 317

Lagage, P. O., \& Cesarsky, C. J. 1983, A\&A, 125, 249

Levesque, E. M. 2011, arXiv:1101.4418

Levesque, E. M., Kewley, L. J., Berger, E., \& Jabran Zahid, H. 2010, AJ, 140, 1557

Levinson, A., \& Eichler, D. 1993, ApJ, 418, L386

Maurin, D., Donato, F., Taillet, R., \& Salati, P. 2001, ApJ, 555, 585

Medvedev, M. V., \& Loeb, A. 1999, ApJ, 526, 697

Pierre AUGER Collaboration, et al. 2010, Phys. Lett. B, 685, 239
Pierre AUGER Collaboration, et al. 2011, Astropart. Phys., 34, 627

Pohl, M., \& Esposito, J. A. 1998, ApJ, 507, 327

Pohl, M., Perrot, C., Grenier, I., \& Digel, S. 2003, A\&A, 409, 581

Pohl, M., \& Schlickeiser, R. 2000, A\&A, 354, 395

Schlickeiser, R. 1989, ApJ, 336, 243

Strong, A. W., \& Moskalenko, I. V. 1998, ApJ, 509, 212

The High Resolution Fly's Eye Collaboration, et al. 2009, Astropart. Phys., 32, 53

Wang, X.-Y., Liu, R.-Y., \& Aharonian, F. A. 2011, ApJ, 736, 112

Wick, S. D., Dermer, C. D., \& Atoyan, A. 2004, Astropart. Phys., 21, 125 